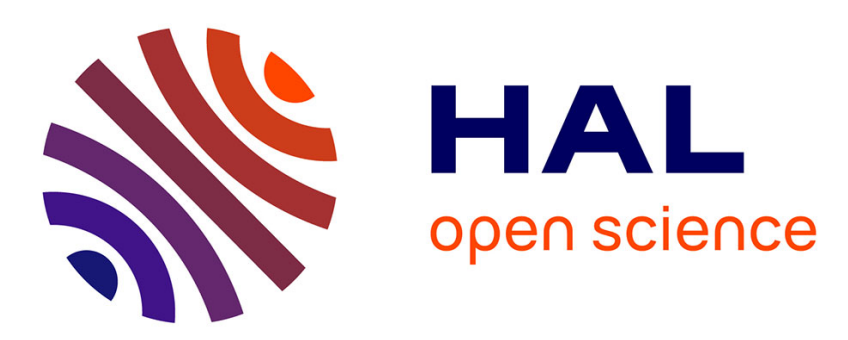

\title{
Investigation of the (100) Surface of the Ce3Pd20Si6 Intermetallic Cage Compound
}

F. Abdel-Hamid, M.-C. de Weerd, J. Ledieu, É. Gaudry, V. Fournée

\section{To cite this version:}

F. Abdel-Hamid, M.-C. de Weerd, J. Ledieu, É. Gaudry, V. Fournée. Investigation of the (100) Surface of the Ce3Pd20Si6 Intermetallic Cage Compound. Journal of Physical Chemistry C, 2019, 123 (19), pp.12355-12366. 10.1021/acs.jpcc.9b02179 . hal-02122057

\section{HAL Id: hal-02122057 \\ https://hal.science/hal-02122057}

Submitted on 29 Nov 2020

HAL is a multi-disciplinary open access archive for the deposit and dissemination of scientific research documents, whether they are published or not. The documents may come from teaching and research institutions in France or abroad, or from public or private research centers.
L'archive ouverte pluridisciplinaire HAL, est destinée au dépôt et à la diffusion de documents scientifiques de niveau recherche, publiés ou non, émanant des établissements d'enseignement et de recherche français ou étrangers, des laboratoires publics ou privés. 


\title{
Investigation of the (100) Surface of the $\mathrm{Ce}_{3} \mathbf{P d}_{20} \mathrm{Si}_{6}$ Intermetallic Cage Compound
}

\author{
F. Abdel-Hamid, M.-C. de Weerd, J. Ledieu, É. Gaudry, ${ }^{*}$ and V. Fournée* \\ Institut Jean Lamour (UMR 7198 CNRS-Nancy-Université de Lorraine), Campus ARTEM \\ - 2 allée André Guinier, BP 50840, F-54011 Nancy, France \\ E-mail: emilie.gaudry@univ-lorraine.fr; vincent.fournee@univ-lorraine.fr
}




\begin{abstract}
Intermetallic cage compounds have crystal structures characterized by three-dimensional frameworks of host atoms forming cages that may be filled with guest atoms. They can be considered as a class of complex metallic alloys (CMA), a broad family of crystalline materials having large or giant unit cells in which a cluster substructure exists. Their bulk physical properties have attracted interest in recent years, particularly as several of them are considered as promising thermoelectric materials. As a different type of CMAs, they may exhibit interesting surface properties which have not been explored until now. Here, we report a detailed investigation of the (100) surface of the $\mathrm{Ce}_{3} \mathrm{Pd}_{20} \mathrm{Si}_{6}$ compound and describe its relationship with the three-dimensional cage framework. The study has been carried out using both experimental surface science methods under ultrahigh vacuum conditions and theoretical calculations based on the Density Functional Theory (DFT). After annealing at moderate temperatures, the surface exhibits two different types of terminations, one of them being a metastable $(2 \times 2)$ reconstruction. Annealing at higher temperatures leads to a single termination with a (1x1) structure. Twelve different surface models have been considered for DFT calculations. The surface phase diagram deduced from first principles calculations with the chemical potentials as variables provides insight into the relative stability of the different surface models. Combining experimental and theoretical results leads to the conclusion that the surface forms at corrugated planes of the bulk structure that preserve intact the $\mathrm{Pd}_{12} \mathrm{Si}_{6}$ cages, with additional $\mathrm{Pd}$ surface atoms. It is concluded that despite this Pd-based cage compound being clearly metallic, covalent-like interactions between $\mathrm{Pd}$ and $\mathrm{Si}$ atoms on the host cage structure significantly influences its surface structure.
\end{abstract}

\title{
Introduction
}

Intermetallic cage compounds have recently attracted interest as a new class of potential thermoelectric materials. ${ }^{1}$ Their crystal structure is characterized by three-dimensional frame- 
works of host atoms forming cages. These cavities are usually filled with guest atoms and the rattling of these atoms damp the phonon transport leading to very low thermal conductivities. ${ }^{2-4}$ They can also have a metallic character, thus fulfilling the phonon glass - electron crystal concept. The $\mathrm{Ce}_{3} \mathrm{Pd}_{20} \mathrm{Si}_{6}$ compound belongs to this class of intermetallics and is also identified as an heavy-fermion system. ${ }^{5}$ The host structure consists of $\mathrm{Pd} / \mathrm{Si}$ cages that are filled by Ce guest atoms. ${ }^{6}$ This phase has interesting physical properties that are partly due to the hybridization between the localized Ce $4 \mathrm{f}$ electrons and the conduction electrons. ${ }^{7-10}$ The temperature dependence of the electrical resistivity is characteristic of a Kondo system, with a maximum observed at a Kondo temperature $\mathrm{T}_{K}$ of about $20 \mathrm{~K}$. Both Ce atom types are in a $\mathrm{Ce}^{3+}$ state at high temperatures $(\geq 100 \mathrm{~K}) .{ }^{10}$ Several studies also reported several magnetic transitions occurring at very low temperatures as well as magnetic field induced quantum criticality. ${ }^{9,11}$

The $\mathrm{Ce}_{3} \mathrm{Pd}_{20} \mathrm{Si}_{6}$ compound is a face-centered cubic crystal (space group $F m \overline{3} m$. The experimental lattice parameter varies between $12.16 \AA^{6}$ and $12.28 \AA,{ }^{5,7}$ associated with a certain homogeneity range around the exact stoechiometric composition due to a partial substitution of $\mathrm{Pd}$ by $\mathrm{Si}$ atoms as reported in. ${ }^{8}$ Its large unit cell contains 116 atoms (12 Ce, $80 \mathrm{Pd}$ and $24 \mathrm{Si}$ atoms). There are 2 inequivalent Ce positions (Ce1(4a) and Ce2(8c)), 2 inequivalent $\mathrm{Pd}$ positions $(\mathrm{Pd} 1(48 \mathrm{~h})$ and $\mathrm{Pd} 2(32 \mathrm{f}))$ and 1 inequivalent Si position $(\mathrm{Si}(24 \mathrm{e}))$, all of them with full occupancy. We use the Wyckoff atomic positions reported in ${ }^{5}$ rather than those reported in ${ }^{6}$ as they were determined for a crystal a lattice parameter $a=12.28$ $\AA$, very close to the experimental lattice parameter of the single crystal used in the present study $(a=12.259 \AA)$. They are listed in table 1 . A change in lattice parameter may reflect a change in stoechiometry and introduce some mixed occupancy of silicon and palladium positions, as well as some variations in interatomic distances, which will not be taken into account in the following. Coordination polyhedra of Ce1 are cubo-octahedral $\mathrm{Pd}_{12} \mathrm{Si}_{6}$ cages and those of $\mathrm{Ce} 2$ are $\mathrm{Pd}_{16}$ cages. The Ce-filled $\mathrm{Pd}_{12} \mathrm{Si}_{6}$ cages form a face-centered sublattice while the $\mathrm{Pd}_{16}$ cages form a simple cubic sublattice inside the crystal. The structure model 
is depicted in Fig. 1.

Table 1: Wyckoff atomic positions in the $\mathrm{Ce}_{3} \mathbf{P d}_{20} \mathrm{Si}_{6}$ crystal $^{5}$

\begin{tabular}{lllll}
\hline \hline Atom & $\mathrm{x}$ & $\mathrm{y}$ & $\mathrm{z}$ & site \\
\hline Ce1 & 0.000 & 0.000 & 0.000 & $4 \mathrm{a}$ \\
$\mathrm{Ce} 2$ & 0.250 & 0.250 & 0.250 & $8 \mathrm{c}$ \\
$\mathrm{Pd} 1$ & 0.000 & 0.168 & 0.168 & $48 \mathrm{~h}$ \\
$\mathrm{Pd} 2$ & 0.382 & 0.382 & 0.382 & $32 \mathrm{f}$ \\
$\mathrm{Si}$ & 0.275 & 0.000 & 0.000 & $24 \mathrm{e}$ \\
\hline \hline
\end{tabular}

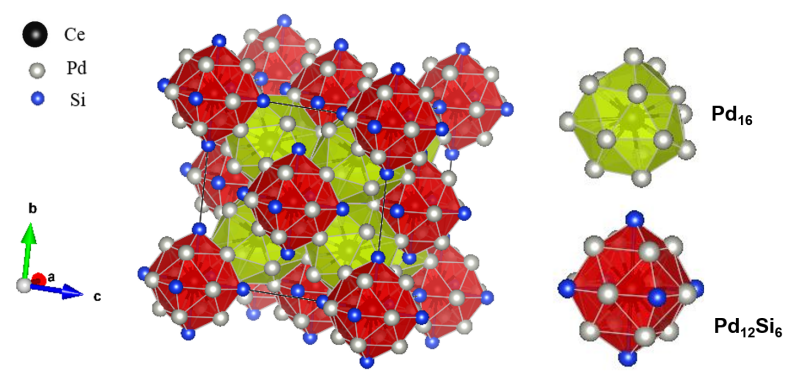

Figure 1: Structure of the $\mathrm{Ce}_{3} \mathrm{Pd}_{20} \mathrm{Si}_{6}$ compound showing the two types of cages $\mathrm{Pd}_{12} \mathrm{Si}_{6}$ and $\mathrm{Pd}_{16}$.

In this paper, we investigate the surface structure of this intermetallic compound and its relationship with the three-dimensional cage framework. The motivation for this work is threefold. First, intermetallic cage compounds can be seen as a class of complex metallic alloys (CMA), based on the definition that CMA represents a broad family of crystalline materials characterized by having large or giant unit cells in which a cluster substructure exists. ${ }^{12}$ It includes complex phases like Samson, Bergman or Zintl phases, and the many CMAs identified following the discovery of quasicrystals. ${ }^{12}$ The surface structures of the latter have been extensively investigated, motivated by both fundamental reasons and potentially useful surface properties (adhesion, friction, oxidation resistance, catalysis). ${ }^{13-15}$ The relationship between the three-dimensional bulk structure and the two-dimensional surface has been questioned in several cases. For example, for both Al-based and Ag-based icosahedral phases, ${ }^{13,16-18}$ it was found that the surface prepared under ultrahigh vacuum 
(UHV) conditions is a bulk termination and consists in flat planes truncating the cluster substructure. In contrast, the surface of the $\mathrm{Al}_{13} \mathrm{Fe}_{4}$ crystalline approximant forms at incomplete puckered planes that exist in the bulk structure. ${ }^{19}$ These highly corrugated planes result from the preservation of the cluster building blocks at the surface, in relation to strong covalent interactions present in this compound. ${ }^{20}$ Intermetallic cage compounds can exhibit similar covalent-like interactions within the framework atoms that may lead to similar phenomena. The surface of these materials have been largely ignored so far, except our recent reports on two low-index surfaces of the $\mathrm{Ba}_{8} \mathrm{Au}_{5.25} \mathrm{Ge}_{40.75}$ phase. ${ }^{21,22}$ In this case, it was found that the cluster substructure dictates the surface morphology. The Ge/Au host cages are preserved at the surface and are stabilized by surface Ba atoms through an electron charge transfer towards the Ge and Au cage atoms, ensuring the saturation of the dangling bonds. The situation might be different for $\mathrm{Ce}_{3} \mathrm{Pd}_{20} \mathrm{Si}_{6}$ because this system is expected to be much more metallic compared to the Ge-based compound. It is nevertheless reasonable to expect that the cage framework could influence the surface structure, leading to an intrinsic surface nanostructuration.

A second motivation to investigate surfaces of intermetallic cage compounds concerns their chemical properties. It is indeed recognized that intermetallics have a great potential in heterogeneous catalysis. ${ }^{23}$ The catalytic performances of a surface depend on both electronic and geometric factors. Because intermetallics have atomic and electronic structures that are fundamentally different from those of their constituent elements, their adsorption and catalytic properties can exhibit a greater variety than those of simple metallic elements or their substitutional alloys. Another advantage of intermetallics is that covalent like interactions can take place, providing a better stability of the active sites under reaction conditions, limiting or excluding segregation phenomena. Promising behavior has been demonstrated for various CMAs including Al-based quasicrystalline approximants ${ }^{23-27}$ but has never been looked at for intermetallic cage compounds. A good knowledge of the surface structure is naturally a prerequisite to understand such properties. 
A third motivation is that Pd-based intermetallics can form hydrides and may be used as hydrogen storage materials with reversible hydrogen adsorption properties. ${ }^{28}$ In $\mathrm{PdH}_{X}$ hydrides, atomic hydrogen occupies octahedral interstices between $\mathrm{Pd}$ atoms. Hydrogen adsorption properties of the $\mathrm{Ce}_{3} \mathrm{Pd}_{20} \mathrm{Si}_{6}$ compound might be interesting to investigate as cavities formed by the $\mathrm{Pd} / \mathrm{Si}$ host atoms may offer large volume for hydrogen storage. This has been demonstrated by a recent theoretical study of silicon-based clathrates, showing that the large interstitial cavities of the host structure can indeed accommodate a large number of hydrogen molecules, equivalent to 10 wt. $\%{ }^{29}$ A good knowledge of the surface structure would again be requested in this perspective.

In the following, we report a detailed investigation of the (100) surface of the $\mathrm{Ce}_{3} \mathrm{Pd}_{20} \mathrm{Si}_{6}$ compound. The experimental and computational methods are presented in part . Experimental results obtained using different surface science techniques are described in part . Section presents the computational results obtained for both the bulk and the surface. All results are combined and discussed in section together with the conclusions.

\section{Methods}

\section{Experimental methods}

A single crystal has been grown in our laboratory using the Czochralski technique. According to the phase diagram, the $\mathrm{Ce}_{3} \mathrm{Pd}_{20} \mathrm{Si}_{6}$ compound is congruent with a melting point $\mathrm{T}_{m} \sim 1523$ K. ${ }^{6,8}$ First, an ingot was prepared by induction melting under a protective Ar atmosphere with a nominal composition equal to $\mathrm{Ce}_{10.3} \mathrm{Pd}_{69.0} \mathrm{Si}_{20.7}$ (in at.\%). Then, the single crystal was pulled from the melt in the Czochralski furnace under Ar atmosphere from a seed crystal oriented along the [111] direction, the preferential growth direction under these experimental conditions. A pulling rate of $0.5 \mathrm{~mm} / \mathrm{h}$ and a rotation speed of $30 \mathrm{rpm}$ was used. The structure of the grown crystal was confirmed by powder X-ray diffraction. The experimental lattice parameter is $a=12.259 \AA$. The crystal had a diameter of $4.5 \mathrm{~mm}$ and was several 
centimeters long. The crystal was then oriented using back-reflection X-ray Laue diffraction and a slice presenting a (100) surface orientation was extracted using a wire saw. The surface of the oriented slice was first prepared by mechanical polishing with decreasing diamond grain size down to $0.25 \mu \mathrm{m}$. Finally, the sample was mounted on a tantalum plate and loaded in the ultrahigh vacuum system with a base pressure in the $10^{-11}$ mbar range. A clean surface was obtained by repeated cycles of $\mathrm{Ar}^{+}$sputtering $(1-2 \mathrm{keV}, 30 \mathrm{~min})$ and subsequent annealing in a temperature range between 723 and $1223 \mathrm{~K}$ for 1 to $2 \mathrm{~h}$. The temperature was measured using an optical pyrometer with an emissivity set to 0.1 as well as by a thermocouple attached on the manipulator. The surface composition and cleanliness was verified by X-ray photoemission spectroscopy (XPS) using a non-monochromatized Mg K $\alpha$ x-ray source. The surface structure was investigated by low-energy electron diffraction (LEED) and scanning tunneling microscopy (STM) at room temperature.

\section{Computational methods}

Spin polarized calculations have been performed within the Density Functional Theory framework using the Vienna ab initio simulation package (VASP code). ${ }^{30-33}$ The interaction between the valence electrons and the ionic core is described using the projector-augmented wave (PAW) method ${ }^{34,35}$ and the calculations are performed within the generalized gradient approximation (GGA-PBE), ${ }^{36,37}$ considering the valence for Ce, Pd and Si to be $[\mathrm{Xe}] 4 \mathrm{f}^{1} 5 \mathrm{~d}^{1}$ $6 \mathrm{~s}^{2},[\mathrm{Kr}] 5 \mathrm{~s}^{1} 4 \mathrm{~d}^{9}$ and [Ne] $3 \mathrm{~s}^{2} 3 \mathrm{p}^{2}$ respectively. The cutoff energy for the plane-wave basis was set to $450 \mathrm{eV}$. Integrations in the Brillouin zone were performed using a $4 \times 4 \times 4 k$-point grid for bulk relaxations such as to achieve an energy precision lower than $1 \mathrm{meV} / \mathrm{at}$. The k-point mesh was increased to $8 \times 8 \times 8$ for bulk density of states calculations. The forceconvergence criterion is $0.02 \mathrm{eV} / \AA$. The convergence parameter for electronic minimization was set to $10^{-5} \mathrm{eV}$.

For the (100) surface, twelve possible models have been built from bulk truncation. We have used symmetric slabs separated by a void thickness comprised between 10 and $17 \AA$. 
The thickness of the slabs is set between 13 and $17 \AA$. The errors related to the previous datasets is estimated to be lower than $10 \mathrm{~mJ} \cdot \mathrm{m}^{-2}$. All atoms in the slab were allowed to relax. STM images have been simulated within the Tersoff-Hamann approximation ${ }^{38,39}$ and plotted at constant current (isodensities in the range $2 \times 10^{-4}$ to $5 \times 10^{-4} \mathrm{e} / \AA^{3}$ ) using the P4VASP software. The surface energies of the considered models have been calculated according to the methods described in Ref. ${ }^{40-45}$ as a function of the chemical potentials of two of the constituting elements, namely $\mathrm{Si}$ and $\mathrm{Pd}$. These can vary within the range $\left(\mu_{i}-\mu_{i}^{\text {bulk }}\right) \in\left[\Delta \mathrm{H}_{f} \times 116 / N_{i} ; 0\right]$, where $\mu_{i}$ and $\mu_{i}^{\text {bulk }}$ are the chemical potentials of element $i$ $(i=\mathrm{Si}, \mathrm{Pd})$ in the slab or in the bulk $\left(N_{S i}=24, N_{P d}=80\right)$ and $\Delta \mathrm{H}_{f}$ is the formation energy of the compound. This leads to numerical values $\Delta \mu_{S i} \in[-3.81 ; 0]$ and $\Delta \mu_{P d} \in[-1.14 ; 0]$ $\mathrm{eV}$ respectively. The Ce chemical potential is constrained by the cohesive energy of the compound $\mu_{C e_{3} P d_{20} S i_{6}}^{\text {bulk }}=3 \mu_{C e}+20 \mu_{P d}+6 \mu_{S i}$.

Bader charge analysis has been performed on a charge density grid. Charge transfers are calculated with $\Delta Q_{X}=\mathrm{Q}_{X}{ }^{i s o l}-\mathrm{Q}_{X}{ }^{\text {slab }}$ with $\mathrm{Q}_{X}{ }^{\text {isol }}$ being the number of electrons for

an isolated atom $\mathrm{X}$ and $\mathrm{Q}_{X}{ }^{\text {slab }}$ the number of electrons for an atom $\mathrm{X}$ in the slab. ${ }^{46-49}$ Calculations of the Electron Localization Function (ELF) are based on valence densities to qualitatively highlight the presence of dangling bonds at the surface. ${ }^{50-54}$ Atomic structures and ELF isodensities were plotted using VESTA software. ${ }^{55}$

\section{Experimental results}

The near surface composition of the $\mathrm{Ce}_{3} \mathrm{Pd}_{20} \mathrm{Si}_{6}(100)$ surface has been measured by XPS as a function of the annealing temperature as well as a function of the take-off angle of the photoelectrons (measured with respect to the surface plane). The composition is estimated from the area of the Ce $3 \mathrm{~d}_{5 / 2}, \mathrm{Pd} 3 \mathrm{~d}_{5 / 2}$ and $\mathrm{Si} 2 \mathrm{~s}$ core-level peaks. To this end, the $\mathrm{Mg}$ K $\alpha$ satellites have been removed and a Shirley background has been subtracted from the core-level spectra. As can be seen in Fig. 2a, there is a small decrease of the Pd content 
(from 71 to 64 at.\%) and a corresponding increase of the Ce content (from 5 to 12 at.\%) with increasing annealing temperature, from 673 to $1173 \mathrm{~K}$. The uncertainty of those measurements are typically estimated at \pm 5 at. $\%$. The Si content is almost constant within this temperature range. The surface concentration measured after annealing at $1023 \mathrm{~K}$ is independent of the take-off angle - and thus of the escape depth of the photoelectrons (see Fig. 2b). The measured composition is $\mathrm{Ce}_{9} \mathrm{Pd}_{69.5} \mathrm{Si}_{21.5}$, i.e. very close to the nominal composition $\mathrm{Ce}_{10.3} \mathrm{Pd}_{69.0} \mathrm{Si}_{20.7}$. From these measurements, it is concluded that the surface concentration is similar to that of the bulk within the accuracy of XPS and that there is no drastic chemical segregation in the near surface region.
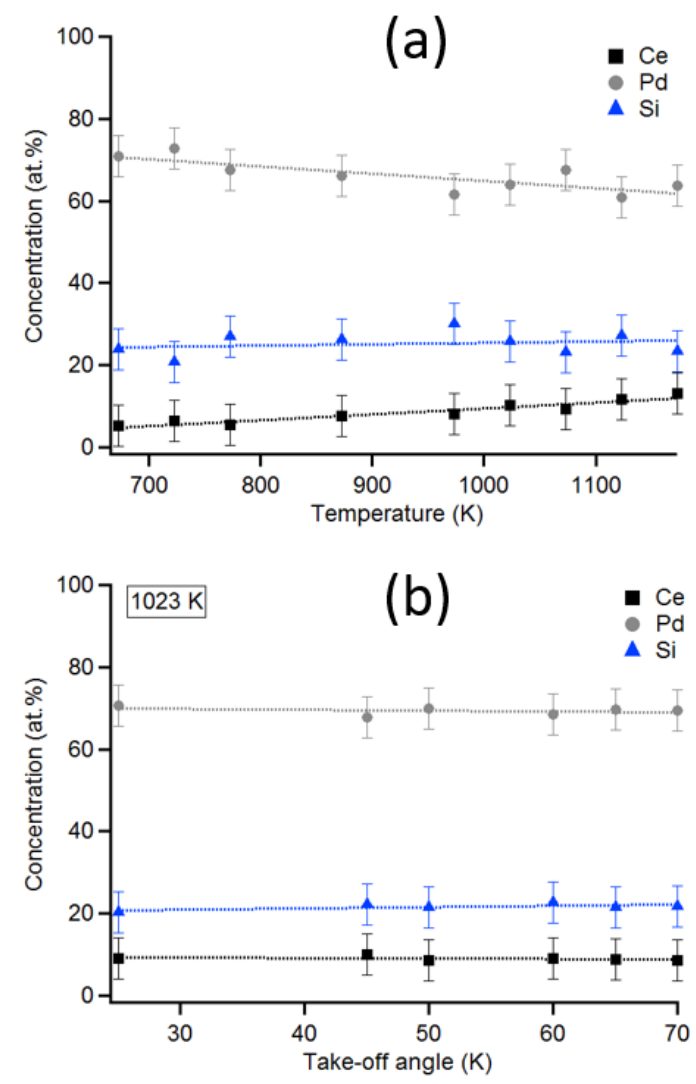

Figure 2: (a) Variation of the chemical concentration of the $\mathrm{Ce}_{3} \mathrm{Pd}_{20} \mathrm{Si}_{6}(100)$ surface measured by XPS as a function of the annealing temperature. (b) Variation of the chemical concentration as a function of the take-off angle after annealing the sample at $1023 \mathrm{~K}$. The dotted lines are linear fit to the data and only guides for the eyes. 


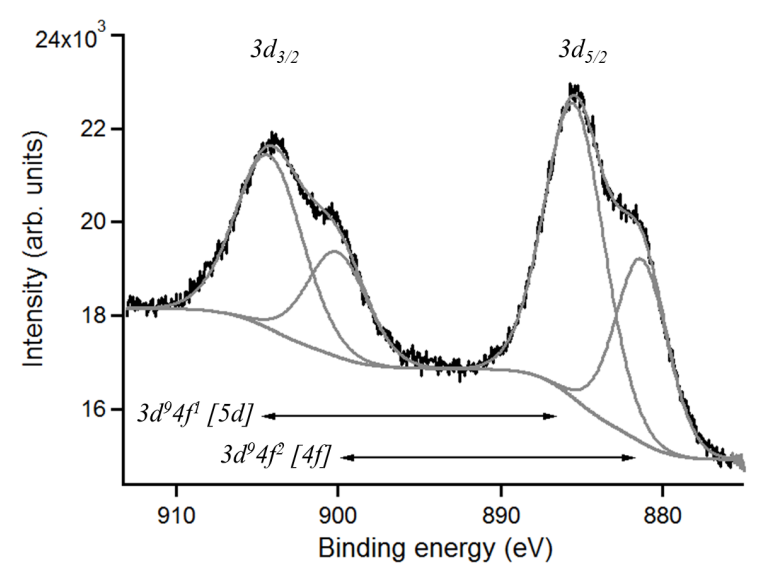

Figure 3: XPS Ce 3d core-level peaks showing satellite peaks.

The Ce $3 d$ core-level peaks are shown in Fig. 3. In addition to the main $3 d_{5 / 2}$ and $3 d_{3 / 2}$ components, there is a clear satellite doublet on the low binding energy side which is attributed to a final state effect arising from two different screening mechanisms of the core hole due to hybridization between the $f$ and $d$ levels. The main peaks are usually ascribed to $5 d$-screening with final state configuration $3 d^{9} 4 f^{1}[5 d]$ while the satellite is associated to $3 d^{9} 4 f^{2}$ [ $\left.4 f\right]$ final state configuration. ${ }^{56}$ The presence of the satellite peaks indicates that there are some Ce $4 f$ states in the valence band close to Fermi level.

Low-energy electron diffraction patterns are observed for sample annealed at a temperature equal to or above $723 \mathrm{~K}$ and up to the highest temperatures used in the present study (1223 K, 0.8.Tm). Two different regimes can be distinguished. Above $823 \mathrm{~K}$, a simple (1x1) pattern consistent with a bulk terminated surface is obtained (Fig. 4a). For annealing temperatures between 723 and $823 \mathrm{~K}$, additional spots consistent with a (2x2) surface reconstruction are observed (Fig. 4b). The momentum space has been calibrated using diffraction patterns of a known crystalline structure recorded under identical experimental conditions. It leads to a square unit mesh with dimensions $a_{1}=a_{2}=8.6 \pm 0.1 \AA \sim a / \sqrt{2}$ for the $(1 \mathrm{x} 1)$ surface and $a_{1 R}=a_{2 R}=17.0 \pm 0.2 \AA$ for the reconstructed lattice of the $\mathrm{Ce}_{3} \mathrm{Pd}_{20} \mathrm{Si}_{6}(100)$ surface.

The surface was investigated by STM and it shows a terrace and step morphology. For annealing temperatures up to $823 \mathrm{~K}$, two different types of terraces are observed (Fig. 5a), 

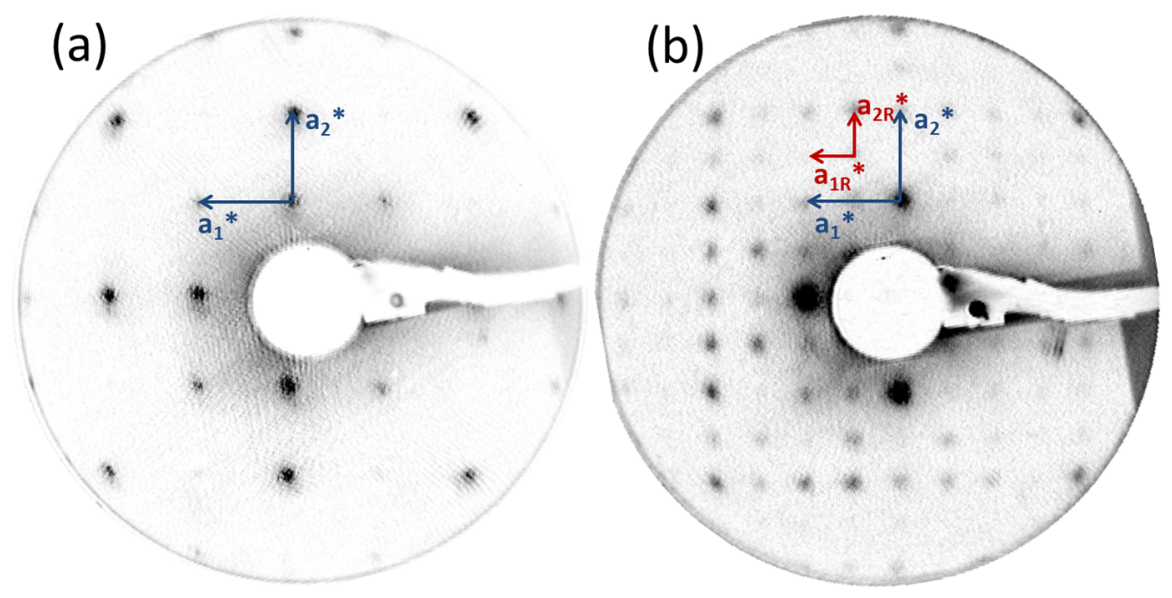

Figure 4: LEED patterns of the $\mathrm{Ce}_{3} \mathrm{Pd}_{20} \mathrm{Si}_{6}(100)$ surface recorded at a primary beam energy of $37 \mathrm{eV}$ after annealing at $873 \mathrm{~K}(\mathrm{a})$ and $773 \mathrm{~K}(\mathrm{~b})$, showing the (1x1) and the $(2 \times 2)$ superstructure respectively. The reciprocal lattice vectors are indicated.

separated by a step height of $3.0 \pm 0.2 \AA$ corresponding to approximately $a / 4$. The two different terraces alternate along the surface normal. The lower and upper terraces in Fig. 5 a correspond to a $(1 \mathrm{x} 1)$ termination while the central terrace shows a $(2 \times 2)$ surface unit cell. After annealing the surface at $873 \mathrm{~K}$ or above, the surface exhibits only (1x1) type of terraces (Fig. 5b) separated by a step height of $6.0 \pm 0.2 \AA$ corresponding to $a / 2$. The $(1 \mathrm{x} 1)$ terminations are partly covered by a more disordered layer lying at $1.8 \pm 0.1 \AA$ above the terrace plane (see Fig. 5b). These patches are always attached to the upward step edges. Note that there is a shift between atomic rows of adjacent $(1 \times 1)$ terraces corresponding to half the surface unit cell parameter, as illustrated by the two lines superimposed on the STM image shown in Fig. 5b. This shift is not present between two consecutive (2x2) terraces. High-resolution STM images of both type of terminations are shown in Fig. 5(c,d), together with their corresponding calculated fast Fourier transform (FFT). The (1x1) structure shows only one protrusion with circular shape distributed on a square lattice with parameter 8.8 $\pm 0.3 \AA$ corresponding to $a / \sqrt{2}$. Peak to peak corrugation of line profiles is $0.6 \AA$ between adjacent protrusions and $1.2 \AA$ along the diagonal direction, with little bias dependency. The STM images do not vary significantly with the bias voltage (within -2 to $+2 \mathrm{eV}$ ), except for a weak contrast change. For the $(2 \times 2)$ structure, the STM contrast consists of 
bright protrusions of three types, located at the node of the reconstructed cell (the brighter ones), at the mid-edge position (intermediate brightness) and at the center (the dimer ones) respectively, corresponding to a maximum height difference of $0.8 \AA$. Surface defects are also observed such as adatoms and vacancies. Once the sample has been annealed at 873 K or above, these $(2 \mathrm{x} 2)$ terraces disappear and only the terraces showing the (1x1) lattice remain. Therefore the $(2 \times 2)$ structure appears to be a transient phase whereas the $(1 \times 1)$ structure is the stable surface structure. The fact that the square surface lattice is shifted by half a unit cell between two adjacent (1x1) terraces suggests that these terminations are based upon bulk layers comprising the $\mathrm{Pd}_{12} \mathrm{Si}_{6}$ cages which form a face-centered sublattice rather than upon layers comprising the $\mathrm{Pd}_{16}$ cages which form a simple cubic sublattice. Consequently, the $(2 \mathrm{x} 2)$ surface planes appearing in between the $(1 \mathrm{x} 1)$ terraces must be built on layers comprising the $\mathrm{Pd}_{16}$ cages. However, the reconstruction cannot be explained based on the bulk structure. In the following, we concentrate on the $(1 \times 1)$ phase and present DFT calculations to propose a structure model derived from the bulk model that describes these surface terminations.

\section{Computational results}

\section{Bulk calculations}

Lattice parameters and cohesive energies obtained after relaxation of elemental bulk $f c c \mathrm{Pd}$, diamond $\mathrm{Si}$, fcc $\alpha$-Ce phase and of $\mathrm{Ce}_{3} \mathrm{Pd}_{20} \mathrm{Si}_{6}$ are gathered in Tab. 2. The $\alpha$-Ce was selected as it is the stable phase of Ce at low temperature (between 0 and $120 \mathrm{~K}$ ) at ambient pressure. ${ }^{36,57,58}$ The starting lattice parameters were the experimental ones for Ce, Pd and Si. Cohesive energies for simple elements were found in good agreement with the literature. The starting lattice parameter for $\mathrm{Ce}_{3} \mathrm{Pd}_{20} \mathrm{Si}_{6}$ was $12.28 \AA$ and has been taken from Ref. ${ }^{5}$ The lattice parameter after relaxation is $12.39 \AA$ corresponding to a small increase of $0.9 \%$, within the $1 \%$ error usually ascribed to the PBE approximation. ${ }^{59}$ 

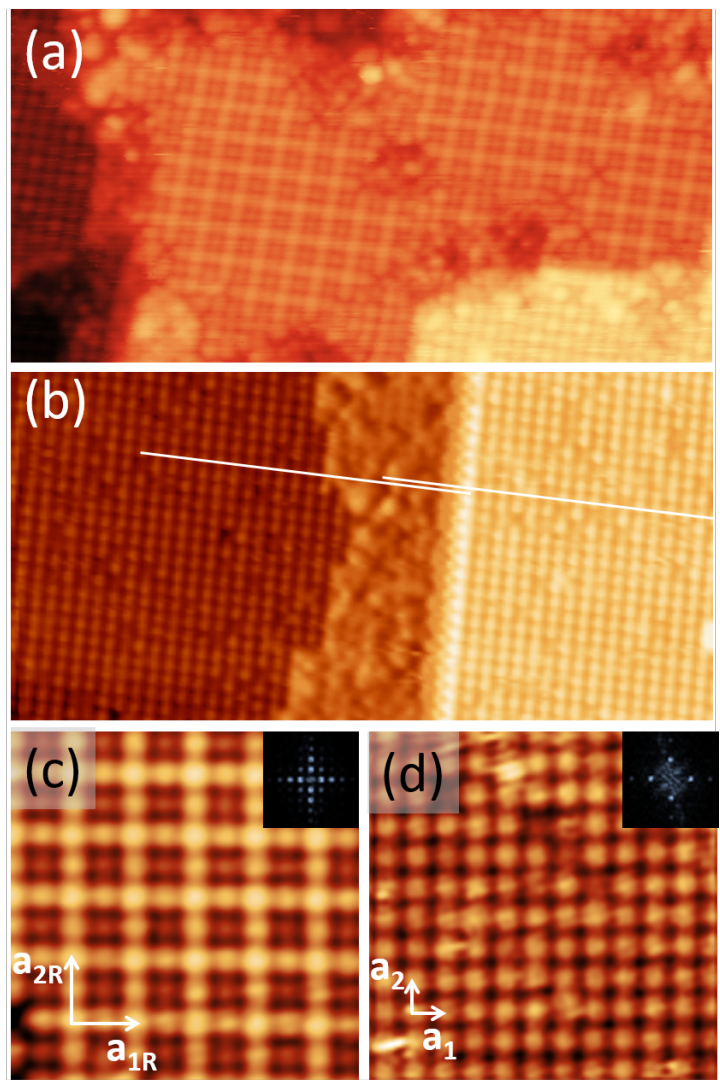

Figure 5: (a,b) $40 \times 20 \mathrm{~nm}^{2} \mathrm{STM}$ images of the $\mathrm{Ce}_{3} \mathrm{Pd}_{20} \mathrm{Si}_{6}(100)$ surface annealed at $773 \mathrm{~K}$ (a) and $873 \mathrm{~K}(\mathrm{~b})$ respectively $\left(V_{b}=+2 \mathrm{~V} ; \mathrm{I}_{t}=0.1 \mathrm{nA}\right)$. Both $(1 \mathrm{x} 1)$ and $(2 \mathrm{x} 2)$ terminations coexist in (a) while only (1x1) termination remains in (b). (c,d) $10 \times 10 \mathrm{~nm}^{2}$ STM images of the $(2 \times 2)$ and (1x1) terminations. The corresponding calculated fast Fourier transforms are shown in the insets.

The calculated cohesive energy is $4.80 \mathrm{eV} /$ at and the formation enthalpy is $\Delta \mathrm{H}=-0.80$ $\mathrm{eV} /$ at. The bulk electronic density of states (DOS) has been calculated. The total DOS is shown in Fig. 6 together with the main contributions from $\mathrm{Ce} f, \mathrm{Pd} d$ and $\mathrm{Si} p$ orbitals. The valence band is dominated by the $\mathrm{Pd} 4 d$ states while Ce $4 f$ states are the main contributors to the conduction band. The Si $s p$ states also contribute weakly to the DOS. These results are in good agreement with fully relativistic calculations reported in Ref. ${ }^{10}$ Note that there are $4 f$ states below the Fermi level, consistent with the final state satellites observed by XPS and in agreement with Ce $4 d-4 f$ resonance photoemission spectroscopy measurements reported in. ${ }^{10}$ The electronic specific heat coefficient estimated from the total DOS at $\mathrm{E}_{F}$ gives $\sim 0.037 \mathrm{~J} / \mathrm{mol} . \mathrm{K}^{2}$. The spin- and site-resolved DOS of $\mathrm{Pd}$ and Ce atoms are shown in 
Table 2: Lattice parameters and corresponding cohesive energies for for $\alpha$-Ce fcc, Pd $f c c, \mathrm{Si}$ diamond and $\mathrm{Ce}_{3} \mathbf{P d}_{20} \mathrm{Si}_{6}$. This work (exp.) indicates that the cohesive energy has been calculated using the experimental lattice parameter. This work (relax.) indicates that the cohesive energy has been calculated using lattice parameter obtained after volume relaxation. 'Experimental' gives experimental values when available. Other lines report previously published values when available. Ref: a: ${ }^{60}$ b: ${ }^{61}$ c:,${ }^{62}$ $\mathrm{d}:{ }^{58}$ e:, ${ }^{63} \mathrm{f:},{ }^{64} \mathrm{g:},{ }^{65} \mathrm{h:},{ }^{66} \mathrm{i:}{ }^{67}$ and $\mathrm{j}:{ }^{5,7}$

\begin{tabular}{|c|c|c|}
\hline Systems and methods & $\mathrm{a}(\stackrel{\circ}{A})$ & $\mathrm{E}_{c o h}(\mathrm{eV})$ \\
\hline \multicolumn{3}{|l|}{$\alpha-\operatorname{Ce} f c c$} \\
\hline This work (exp.) & 4.83 & 4.61 \\
\hline This work (relax.) & 4.52 & 4.33 \\
\hline Experimental & $4.83^{a}, 4.85^{b}$ & $4.32^{c}$ \\
\hline LDA & $4.52^{b}, 4.60^{d}$ & $5.84^{d}$ \\
\hline GGA-PBE & $4.74^{b}, 4.68^{d}$ & $4.93^{d}$ \\
\hline \multicolumn{3}{|l|}{$\mathrm{Pd} f c c$} \\
\hline This work (exp.) & 3.89 & 3.75 \\
\hline This work (relax.) & 3.94 & 3.77 \\
\hline Experimental & $3.89^{e}$ & $3.90^{e}, 3.89^{c}$ \\
\hline LDA & $3.84^{f}$ & - \\
\hline GGA-PBE & $3.95^{e}, 3.94^{f}$ & $3.71^{e}$ \\
\hline \multicolumn{3}{|l|}{ Si diamond } \\
\hline This work (exp.) & 5.43 & 4.56 \\
\hline This work (relax.) & 5.47 & 4.57 \\
\hline Experimental & $5.43^{g}, 5.41^{e}$ & $4.63^{c}, 4.62^{h}$ \\
\hline LDA & $5.40^{e}, 5.43^{h}$ & $5.26^{h}$ \\
\hline GGA-PBE & $5.47^{e, i}, 5.49^{h}$ & $4.50^{h}, 4.56^{i}$ \\
\hline \multicolumn{3}{|l|}{$\mathrm{Ce}_{3} \mathrm{Pd}_{20} \mathrm{Si}_{6}$} \\
\hline This work (exp.) & $12.28^{j}$ & 4.80 \\
\hline This work (relax.) & 12.39 & 4.800 \\
\hline
\end{tabular}

Fig. 7. The partial DOS of Pd are similar for both spin up and spin down polarizations for the two different crystallographic sites, consistent with the absence of calculated magnetic moment carried by these atoms. The partial DOS of Ce shows spin polarization at the (8c) sites (Ce2) but not at (4a) sites (Ce1), again in agreement with the finding that the magnetic properties of the $\mathrm{Ce}_{3} \mathrm{Pd}_{20} \mathrm{Si}_{6}$ compound arise from the (8c) sites only. ${ }^{68}$ The $f$ states at (4a) sites are also more localized and contribute less at the Fermi level than the $f$ states at $(8 \mathrm{c})$ sites. All these results are in reasonable agreement with those previously reported in, ${ }^{10}$ except for the exact shape of the partial DOS of Ce. This may be due to the fact that 
we used a plane-wave calculation method (not an all-electron method) and that relativistic effects have only been partially taken into account in the present work.

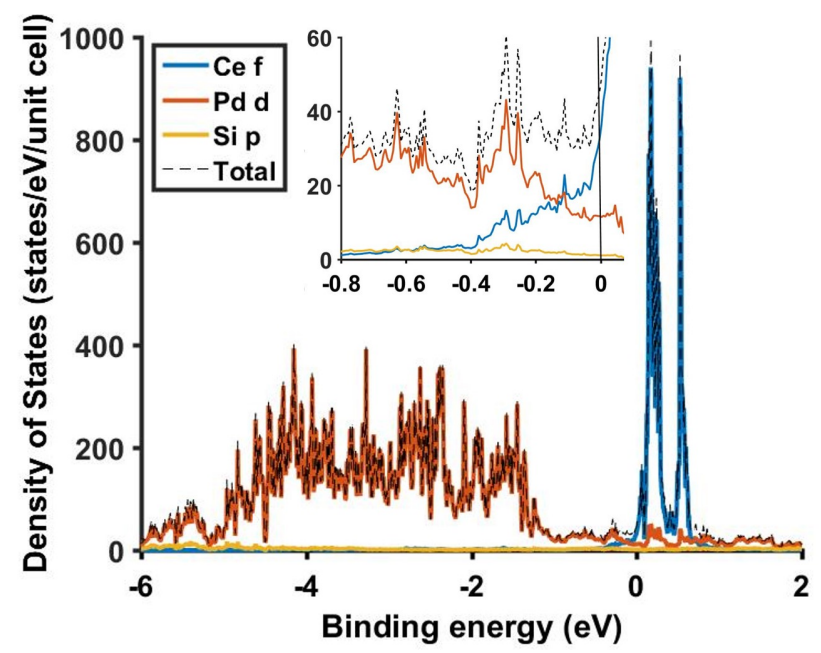

Figure 6: Calculated total DOS of the bulk $\mathrm{Ce}_{3} \mathrm{Pd}_{20} \mathrm{Si}_{6}$ compound together with the main contributions from $\mathrm{Ce} f, \operatorname{Pd} d$ and $\mathrm{Si} p$ orbitals. The inset shows a zoom on these partial DOS in the vicinity of the Fermi level.

\section{Surface models}

Twelve possible surface models were built from bulk truncation, some preserving the cage substructure and others truncating these clusters. These models are illustrated in Fig. 8, showing both top and side views.

Model 1 completely preserves the $\mathrm{Pd}_{12} \mathrm{Si}_{6}$ cages (red cages). Model 2 is obtained from model 1 by adding $\mathrm{Pd} 2$ atoms belonging to the $\mathrm{Pd}_{16}$ cages located at $1.45 \AA$ above the origin layer $(\mathrm{X}=0)$ as shown in Fig. 9a (there are $8 \mathrm{Pd} 2$ atoms per unit cell). Model 3 is obtained from model 1 by removing Si topmost atoms (2 Si atoms per unit cell). Model 4 can be obtained by removing Si topmost atoms from model 2 or equivalently by adding the same $\mathrm{Pd} 2$ atoms to model 3. Model 5 is obtained by truncating the $\mathrm{Pd}_{12} \mathrm{Si}_{6}$ cages in their equatorial plane, i.e. at the origin layer $(\mathrm{X}=0)$. If the $\mathrm{Pd} 2$ atoms are conserved, then model 6 is obtained. Model 7 is the one preserving the $\operatorname{Pd}_{16}$ cages. The layer of topmost $\mathrm{Pd}$ atoms of model 7 is the same of that obtained from model 5 after removing both $\mathrm{Ce} 2$ and $\mathrm{Si}$ atoms. 


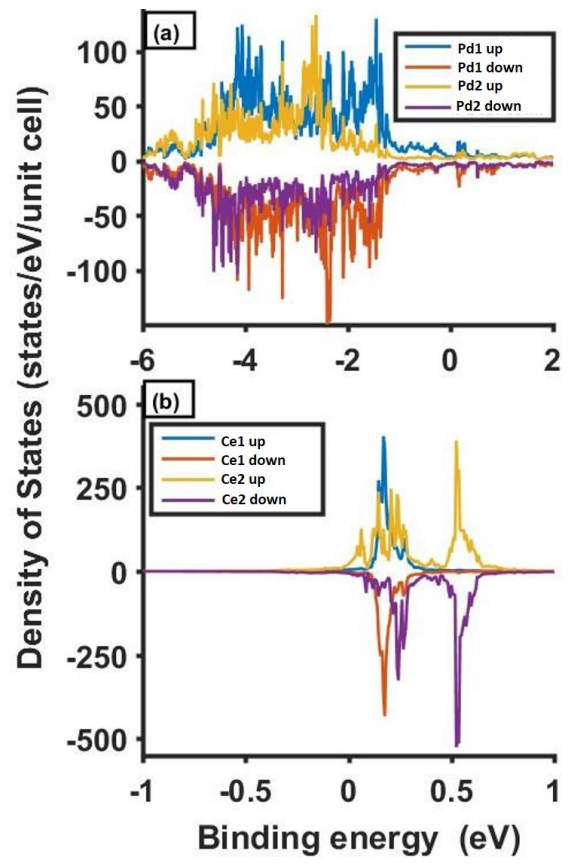

Figure 7: Spin- and site-resolved DOS for $\mathrm{Pd}$ and Ce atoms in the bulk $\mathrm{Ce}_{3} \mathrm{Pd}_{20} \mathrm{Si}_{6}$ compound.

Model 8 is obtained from model 7 by removing this layer of topmost $\mathrm{Pd}$ atoms, exposing the $\operatorname{Pd} 2$ atoms (at $4.69 \AA$ ) at the surface. Model 8 thus intercepts the $\operatorname{Pd}_{16}$ cages. Removing these Pd2 atoms from model 8 leads to model 9 which is formed by a layer of $\operatorname{Pd} 1$ atoms located at $4.08 \AA$. If this layer is removed again, then $\mathrm{Ce} 1$ atoms occupying the $\operatorname{Pd}_{16}$ cluster centers located at $3.07 \AA$ are present at the surface. These leads to models 10 to 12 , which differ by the number of Si atoms preserved at the surface. Model 12 is obtained if Si atoms located at both $3.38 \AA$ and $2.76 \AA$ are maintained, model 11 is obtained if only the two Si atoms per unit cell located at $2.76 \AA$ are maintained (the one that are attached to $\mathrm{Pd}_{12} \mathrm{Si}_{6}$ cages located above). When both types of Si atoms are removed, one obtains model 10. The relationship between the various models is schematically illustrated in Fig.9b.

These models are representatives of all possible models which can be built from bulk truncation. They differ by their atomic structure, their chemical composition and intrinsic roughness, and preserve - or not - the cage integrity. Tab.3 indicates for all models the number and types of atoms and the atomic density $\rho$ given in at. $/ \mathrm{nm}^{3}$ calculated by counting all atoms within a $3 \AA$ thick flat strip intercepting the topmost surface atoms (where 3 

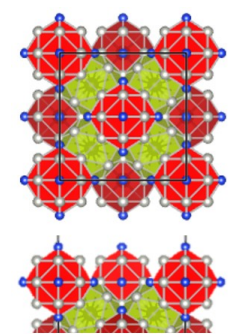

(M1)
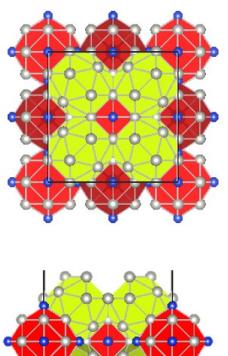

(M7)
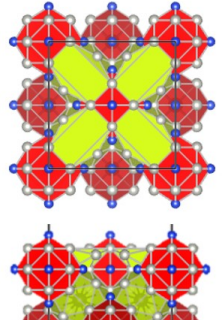

(M2)
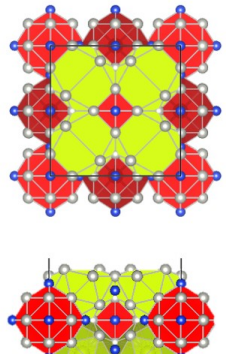

(M8)
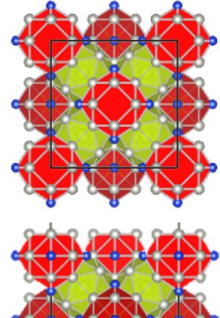

(M3)
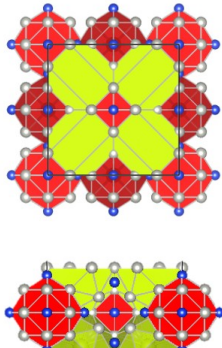

(M9)
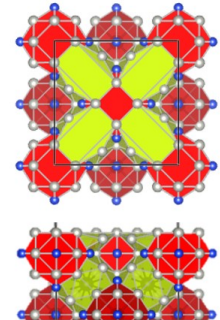

(M4)
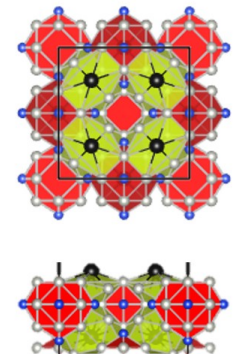

(M10)
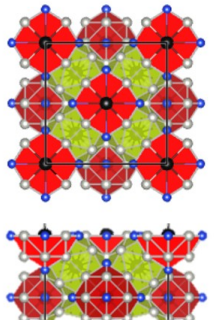

(M5)
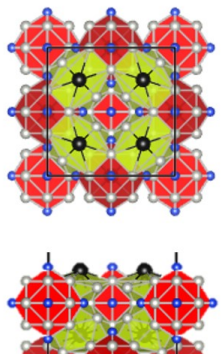

(M11)
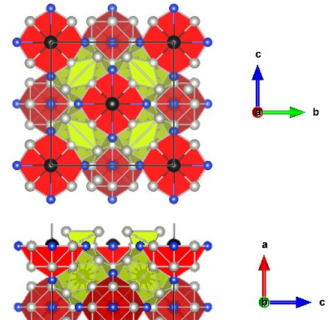

(M6)
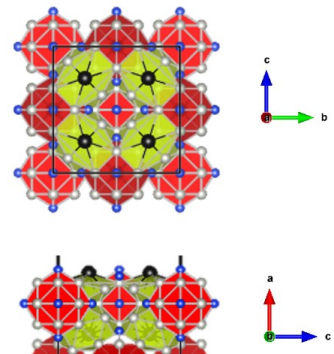

(M12)

Figure 8: Crystallographic structure of the various surface models. For each model, the top part shows the top view, the bottom part shows the side view. In top views, the topmost cages are illustrated with a slightly darker color. Ce, Pd and Si atoms are represented by black, gray and blue circles respectively.

(a)

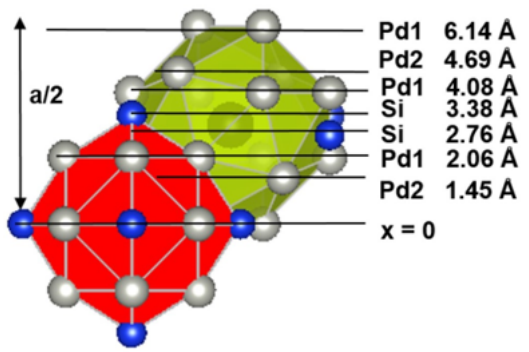

(b)

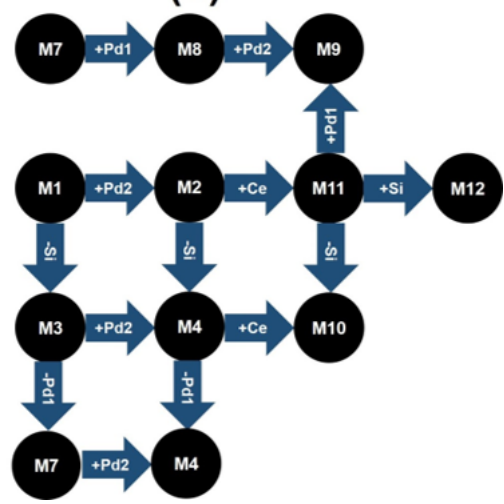

Figure 9: (a) Atomic structure of one $\mathrm{Pd}_{12} \mathrm{Si}_{6}$ and one $\mathrm{Pd}_{16}$ cages extracted from unit cell, (b) Schematic illustrating the relationships between the various models. 
$\AA$ corresponds to the average surface roughness).

Table 3: Atomic composition of the various surface models and corresponding atomic density $\rho$ given in $a t / \mathrm{nm}^{3}$. These are calculated by counting all atoms per surface unit cell within a $3 \AA$ thick flat strip intercepting the topmost surface atoms.

\begin{tabular}{lllllll}
\hline \hline Model & $\mathrm{P}$ or $\mathrm{F}$ & $\mathrm{N}_{C e}$ & $\mathrm{~N}_{P d}$ & $\mathrm{~N}_{S i}$ & $\mathrm{~N}_{\text {total }}$ & $\rho$ \\
\hline M1 & $\mathrm{P}$ & 5 & 20 & 17 & 42 & 82.5 \\
M2 & $\mathrm{P}$ & 5 & 28 & 17 & 50 & 98.2 \\
M3 & $\mathrm{P}$ & 5 & 23 & 12 & 40 & 78.5 \\
M4 & $\mathrm{P}$ & 5 & 29 & 12 & 46 & 90.3 \\
M5 & $\mathrm{F}$ & 9 & 28 & 19 & 56 & 109.9 \\
M6 & $\mathrm{P}$ & 5 & 24 & 14 & 43 & 84.4 \\
M7 & $\mathrm{P}$ & 5 & 28 & 8 & 41 & 80.5 \\
M8 & $\mathrm{P}$ & 5 & 38 & 6 & 49 & 96.2 \\
M9 & $\mathrm{P}$ & 5 & 32 & 6 & 43 & 84.4 \\
M10 & $\mathrm{P}$ & 9 & 20 & 6 & 35 & 68.7 \\
M11 & $\mathrm{P}$ & 9 & 26 & 17 & 52 & 102.1 \\
M12 & $\mathrm{P}$ & 9 & 26 & 21 & 57 & 111.9 \\
\hline \hline
\end{tabular}

\section{Simulated STM images}

STM images have been calculated within the Tersoff-Hamman approximation using the constant current mode ${ }^{69}$ where the tunneling current I is proportional to the local density of states at the tip position. The simulated images for all models are shown in Fig. 10 at positive and negative bias $(+1$ and $-1 \mathrm{~V})$.

For most of the models, the STM contrast consists in bright features located at the node and at the center of the surface unit cell. These bright features arise not from individual atoms but rather from groups of atoms. The bias dependency is also more or less pronounced depending on the models. For example for the model 1, the bright features have a rounded shape and they arise from $\mathrm{Pd}$ and $\mathrm{Si}$ atoms forming the top part of the $\mathrm{Pd}_{12} \mathrm{Si}_{6}$ cages protruding at the surface. No bias dependency is observed in this case. The maximal brightness at the center of the spots correspond to the topmost Si atoms. Some intensity is observed connecting the cages separated by a distance of $a / \sqrt{2}$, arising from Pd1 type of atoms belonging to adjacent cages. A similar pattern is found for model 2 calculated for the 
same isodensity but it shows a larger contrast variation in between the bright dots as well as a small bias dependency. When topmost $\mathrm{Si}$ atoms are removed from the protruding $\mathrm{Pd}_{12} \mathrm{Si}_{6}$ cages like in models 3 and 4 for example, the rounded bright spots become more square-like shapes tilted by $45^{\circ}$ with respect to the $c$ axis and arising from $4 \mathrm{Pd} 1$ atoms forming a square pattern of side $2.92 \AA$. The vacancy left by the missing $\mathrm{Si}$ atoms can be seen at $\mathrm{V}_{b}=-1$ $\mathrm{V}$ as a small depression at the center of the square motifs. Several models present a similar characteristic, with a more or less pronounced depression at the center of the bright features. Some models exhibit a totally different pattern, like models 7, 10 and 12, for which a dark contrast is observed at the node and at the center of the surface unit cell. For model 7 , the bright features arise from the $\mathrm{Pd}$ atoms forming $\mathrm{Pd}_{16}$ cages protruding at the surface. For model 10, the bright features arise from Ce atoms emerging at the surface by truncating the $\mathrm{Pd}_{16}$ cages. Model 12 contains $\mathrm{Ce}, \mathrm{Si}$ and $\mathrm{Pd}$ surface atoms and is strongly bias dependent.

\section{The surface energy diagram}

To further discriminate between the different models, theIR surface energies have been calculated as a function of the chemical potentials of two of the constituting elements, namely Si and $\mathrm{Pd}$. Each model is represented by a plane in the chemical potentials space. Superimposing the 3D representations of the surface energies for each model allows to determine the surface phase diagram by considering the criterion of the minimum surface energy in every region of the allowed chemical potentials. The constrain on the chemical potential of Ce leads to a forbidden zone in the chemical potentials space as shown in Fig. 11. The diagram takes into account an uncertainty in surface energies of $\delta \gamma=10 \mathrm{~mJ} / \mathrm{m}^{2}$ to distinguish between energetically close models.

The most stable models are model 2 in the Pd and Si-rich limit of the chemical potential, model 10 in the Si-poor limit and model 7 in the Pd-poor limit. Models 4 and 6 also appear at boundaries of the existence domain of model 2. The minima of the surface energy range between $0.2 \mathrm{~J} / \mathrm{m}^{2}$ and $1.4 \mathrm{~J} / \mathrm{m}^{2}$ in the allowed chemical potentials space. The lowest values 


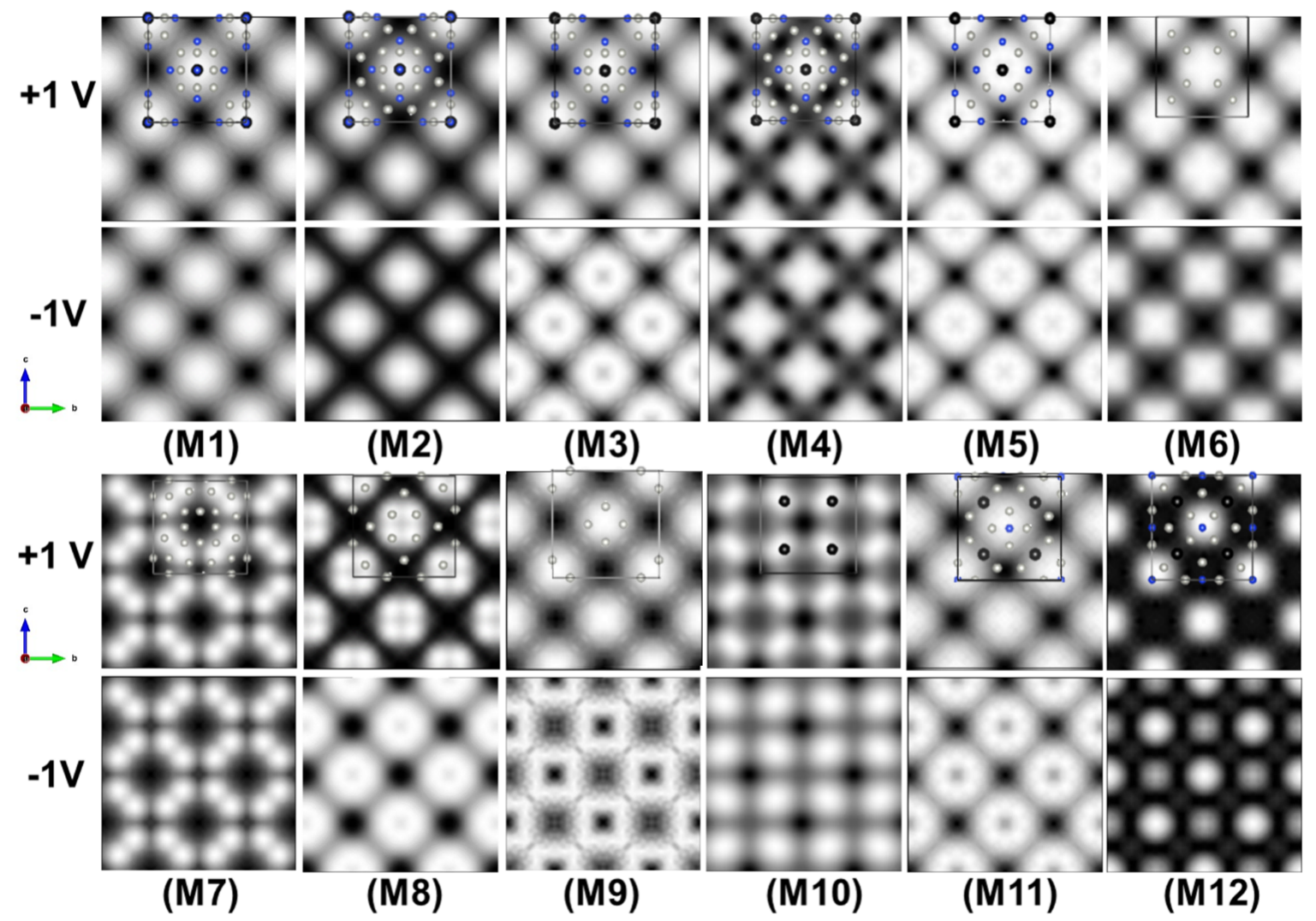

Figure 10: Simulated STM images in constant current mode for the twelve considered models at +1 and $-1 \mathrm{~V}$ bias voltage. The top surface unit cell of the corresponding model is superimposed on the top rows, where $\mathrm{Ce}, \mathrm{Pd}$ and $\mathrm{Si}$ atoms are represented by black, gray and blue circles respectively.

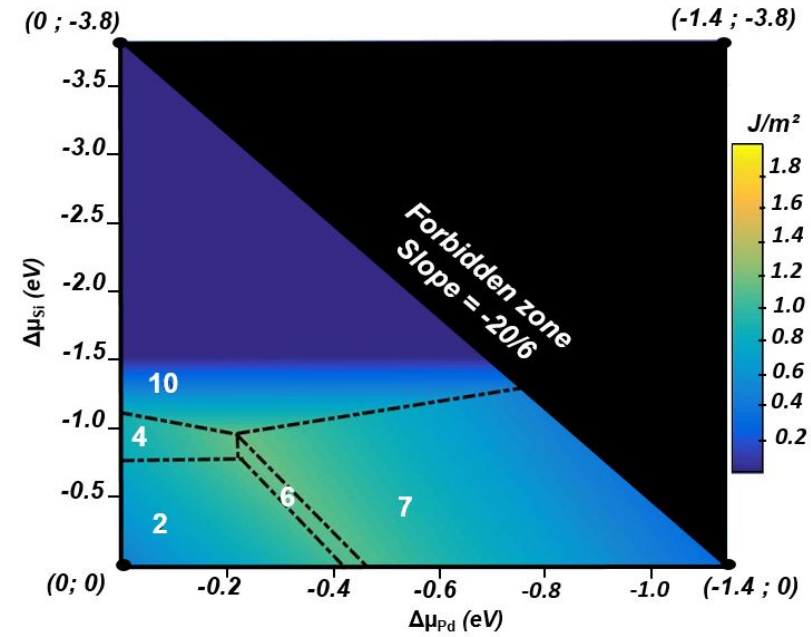

Figure 11: Calculated surface phase diagram for (100) surface. The most stable surface models are numbered in white. The dotted lines indicate the boundary between the most stable models in the chemical potential map. 
are found for model 10 in the Si-poor limit of the chemical potential $\left(0.2 \mathrm{~J} / \mathrm{m}^{2}\right)$, for model 7 in the Pd-poor limit $\left(0.2 \mathrm{~J} / \mathrm{m}^{2}\right.$ to $\left.0.4 \mathrm{~J} / \mathrm{m}^{2}\right)$ and for model 2 in the Pd-rich and Si-rich limit (about $0.4 \mathrm{~J} / \mathrm{m}^{2}$ ). Slightly higher values are found for model 4 and 6 at the boundaries of the existence domain of model 2. Models 7 and 10 are both based on a surface terminating at $\mathrm{Pd}_{16}$ cages, that are either preserved (model 7) or truncated (model 10) and thus exposing Ce atoms in the top surface plane. On the contrary, models 2, 4 and 6 are based on a surface terminating at $\mathrm{Pd}_{12} \mathrm{Si}_{6}$ cages. These models contain more $\mathrm{Si}$ in the near surface region compared to model 7 and 10. Note that the elemental surface energies are 1.09, 1.33 and $1.43 \mathrm{~J} / \mathrm{m}^{2}$ for $\mathrm{Ce}, \mathrm{Si}$ and $\mathrm{Pd}$ respectively (weighted surface energies over the crystal Wulff shape $\left.{ }^{70}\right)$.

\section{Electronic Localization Function and Bader charge analysis}

The electron localization function (ELF) has been calculated to qualitatively highlight electronic effects induced at the surface. The ELF is a normalized function ranging between 0 and 1. The degree of localization increases with increasing ELF value, a magnitude of 0.5 corresponding to a free electron like distribution. The main result is that the calculated ELF shows a lobe structure located on the $\mathrm{Si}$ surface atoms indicative for the presence of a dangling bond. This is shown in Fig. 12(a) for model 2 but is also true for other models having protruding $\mathrm{Si}$ atoms (i.e. models 1, 5, 11 and 12). The Fig. S2 shows the calculated ELF for models 1, 2, 4, 5, 11 and 12 for comparison. One also sees areas of strong localization around the Ce atoms. This is actually a known artifact due the construction of the PAW pseudopotentials in which core electrons are included. ${ }^{71}$ For all other models, ELF shows a delocalized character of the electrons at the surface originating from Pd surface atoms. One also notices that the ELF isosurfaces around $\mathrm{Si}$ atoms significantly depart from a spherical distribution indicating chemical interactions with neighboring Pd atoms. This suggests that covalent-like interactions take place within the $\mathrm{Pd}_{12} \mathrm{Si}_{6}$ cage framework.

The Bader atomic regions are shown in Fig. 12(b) for each atom type. The Bader regions 


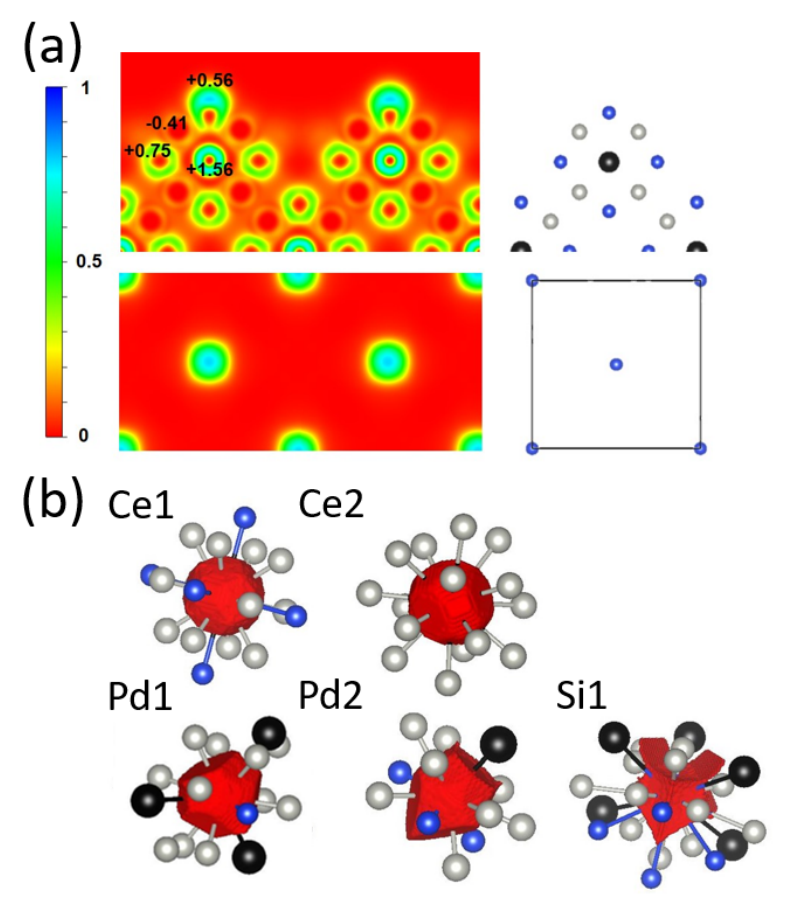

Figure 12: (a) Two-dimensional representation of the electron localization function calculated for the slab model 2 (left). The top part shows a cut through the $(0,0,1 / 2)$ plane perpendicular to the surface and intercepting the protruding $\mathrm{Si}$ atoms and the Ce atoms occupying the $\mathrm{Pd}_{12} \mathrm{Si}_{6}$ cluster centers. Numbers indicate the Bader charges of selected atoms. The bottom part is a cut parallel to the surface and intercepting the top Si atoms. The right part shows the corresponding structure models for the side view (top) and the top view (bottom). (b) Shape of the Bader volumes around each atom type. $\mathrm{Ce}, \mathrm{Pd}$ and $\mathrm{Si}$ atoms are represented by black, gray and blue circles respectively.

around Ce atoms are rather spherical indicating an essentially ionic character. The shape of the Bader volume around $\mathrm{Pd}$ atoms are more polyhedral with flat contact faces between first Pd and Si neighbors. The shape of the Bader volume around Si atoms are even more complex with facets perpendicular to the short Si-Pd2 and Si-Pd1 bonds. These are characteristic of non-polar covalent bonding interactions. The Bader charges have been extracted from the calculated charge densities in the Bader volume around each atomic site for surface atoms as well as for their equivalent position in the bulk (see Tab. 4). It shows that Pd atoms gain on average about $0.42 \mathrm{e} / \mathrm{at}$. in the bulk while $\mathrm{Ce}$ and Si release 1.54 and $0.64 \mathrm{e} / \mathrm{at}$. respectively. This is in agreement with the fact that $\mathrm{Pd}$ is the most electronegative element in the compound (Pauling electronegativity values for $\mathrm{Ce}, \mathrm{Si}$ and $\mathrm{Pd}$ are 1.12, 1.9 and 2.2 
Table 4: Surface Bader charge analysis. The numbers correspond to charge differences $\Delta Q_{X}=\mathbf{Q}_{X}{ }^{i s o l}-\mathbf{Q}_{X}{ }^{\text {slab }}$ with $\mathbf{Q}_{X}{ }^{i s o l}$ being the number of electrons for an isolated atom $\mathbf{X}$ and $\mathbf{Q}_{X}{ }^{\text {slab }}$ the number of electrons for an atom $\mathbf{X}$ at the surface of the slab or in the first subsurface plane where it is present otherwise. The first line gives the average Bader charge for bulk atoms.

\begin{tabular}{cccccc}
\hline \hline Model & Pd1 & Pd2 & Si & Ce1 & Ce2 \\
\hline Bulk & -0.45 & -0.38 & +0.64 & +1.59 & +1.51 \\
M1 & -0.38 & - & +0.68 & +1.52 & - \\
M2 & -0.41 & -0.28 & +0.56 & +1.56 & - \\
M3 & -0.93 & - & +1.25 & +1.51 & - \\
M4 & -0.75 & -1.22 & +2.19 & +1.52 & - \\
M5 & -0.55 & - & +0.50 & +1.60 & - \\
M6 & -0.53 & -0.32 & +0.66 & +1.55 & - \\
M7 & -0.21 & -0.28 & +0.51 & - & +1.55 \\
M8 & -0.31 & -0.38 & $+0.76 /+0.59^{1}$ & - & +1.53 \\
M9 & - & -0.47 & $+0.88 /+0.47$ & - & +1.52 \\
M10 & -0.62 & -0.36 & +0.62 & - & +1.41 \\
M11 & -0.60 & -0.37 & +0.25 & - & +1.43 \\
M12 & -0.61 & -0.61 & $+0.23 /+0.28$ & - & +1.49 \\
\hline \hline
\end{tabular}

respectively $\left.{ }^{72,73}\right)$. The charge transfers for Ce atoms are similar for surface or bulk atoms as can be seen from Tab. 4. The Bader charges of Si and Pd atoms vary significantly with the models. In particular, the charge on $\mathrm{Si}$ atoms located at the tip of the $\mathrm{Pd}_{12} \mathrm{Si}_{6}$ cages in model 1 and 2 remains similar to the corresponding bulk value and the same is true for neighboring $\mathrm{Pd}$ atoms. However when these top Si atoms are removed (leading to model models 3 and 4), the Bader charges increase significantly (up to - 0.93 e/at. for the top $\mathrm{Pd}$ atoms and up to $+2.19 \mathrm{e} / \mathrm{at}$. for neighboring Si atoms), that may contribute to surface destabilization. This is illustrated in Fig. S2 showing two-dimensional representations of the ELF function together with the Bader charges for several surface models. The surface DOS are shown in Fig. S3 for model 2 for Pd1, Pd2 and Si atoms and are compared to the corresponding bulk-like contributions. It shows a metallic character as well as a reduction of the $\mathrm{Pd} d$ band width in relation with the reduced coordination number of Pd surface atoms. There is also an increase of the Si $p$ DOS at the Fermi level compared to the bulk. 


\section{Discussion}

From the experimental section, it is concluded that the $(1 \mathrm{x} 1)$ surface planes are separated by a unique step height of $6.0 \pm 0.2 \AA \sim a / 2$, suggesting that only a specific subset of bulk planes appears as surface termination. A shift of half the surface lattice parameter is observed between adjacent (1x1) terraces, consistent with the fact that these surface terminations form at bulk layers intercepting the $\mathrm{Pd}_{12} \mathrm{Si}_{6}$ cages forming an $f c c$ sublattice. The atomic structure of these planes is not completely resolved by STM as images only reveal bright protrusions distributed on a square lattice with parameter $8.8 \pm 0.3 \AA \sim a / \sqrt{2}$. These bright protrusions most probably do not correspond to single atoms but to groups of atoms. To get further insight into the surface structure, DFT calculations have been performed using twelve possible surface models built from bulk truncation. Some of these models preserve the cage substructure while others truncate these clusters. For the sake of completeness, we have also considered surface models intercepting the bulk structure both at $\mathrm{Pd}_{12} \mathrm{Si}_{6}$ layers and at $\mathrm{Pd}_{16}$ layers. STM images have been simulated for each of the considered models at both positive and negative bias. By comparing with the experimental image shown in Fig. 5 (which do not show significant bias dependency), it is clear that models 7, 9, 10 and 12 can be disregarded because the geometrical motifs are different from the experimental one. The models 3, 5, 8 and 11 show some bias dependency, with a more or less pronounced depression at the center of the bright protrusion at negative bias. Such a feature is either not observed or not resolved in the experimental images. The bright protrusions have a more square appearance in models 4,5 and 6 in comparison with experiments. In addition, model 6 exhibits a significant bias dependence. We mentioned above that the square surface lattice is shifted by half a unit cell between two adjacent (1x1) terraces. This can only be explained if the surface forms at atomic layers preserving or intercepting the $\operatorname{Pd}_{12} \mathrm{Si}_{6}$ cages which form a face-centered cubic sublattice, but would be inconsistent if the surface occurred at atomic layers preserving or intercepting the $\mathrm{Pd}_{16}$ cages which form a simple cubic sublattice. Therefore models 7, 8 and 9 are unlikely. Summarizing all these informations, the 
best match is obtained for models 1 and 2, but we cannot totally exclude models 3, 4, 6 and 11 on that basis. To further discriminate between the models, their surface energies have been calculated as a function of the chemical potentials of two of the constituting elements, namely Si and Pd. The most stable models are model 2 in the Pd and Si-rich limit of the chemical potential, model 10 in the Si-poor limit and model 7 in the $\mathrm{Pd}$-poor limit. Models 4 and 6 also appear at the boundary of the existence domain of model 2 and they have higher surface energies. It is consistent with the fact that these models are variations of model 2 , obtained by removing some of the top Si (M4) plus some top Pd atoms (M6). The lowest surface energy values are found for model 10. However, model 10 as well as model 7 can be safely excluded on the basis of the STM analysis.

Combining experimental data, simulated STM images and calculated surface energies allows to converge towards model 2 as the best model among all models considered here describing the $(1 \mathrm{x} 1)$ surface termination. In this model, the $\mathrm{Pd}_{12} \mathrm{Si}_{6}$ cages are preserved and the protruding Si atoms have a Bader charge that is not modified compared to the corresponding bulk atoms. The surface is further stabilized by additional $\operatorname{Pd} 2$ atoms rendering the surface more compact. These additional $\mathrm{Pd} 2$ atoms have the shortest interatomic distances with the Si atoms $(2.43 \AA)$ and must therefore be strongly bonded. The fact that the surface preserves the $\mathrm{Pd}_{12} \mathrm{Si}_{6}$ cages rather than the $\mathrm{Pd}_{16}$ ones may also be related to stronger bonding between Si and Pd atoms as revealed by the ELF, stabilizing the cage framework. As mentioned in the Introduction, we reported recently a surface study of related intermetallic clathrate, the $\mathrm{Ba}_{8} \mathrm{Au}_{5.25} \mathrm{Ge}_{40.75}$ phase. ${ }^{21,22}$ Although they are both cage compounds, the two systems are quite different in terms of chemistry, one being a Ge-based compound while the other one is Pd-based. In case of $\mathrm{Ba}_{8} \mathrm{Au}_{5.25} \mathrm{Ge}_{40.75}$, the $\mathrm{Au}$-doped $\mathrm{Ge}$ host cages are also preserved at the surface, but additional Ba surface atoms must also be present to stabilize the surface. Numerical simulations indicated that the surface is indeed electronically stabilized by these protruding Ba guest atoms through a charge transfer towards the Ge and Au atoms, saturating the dangling bonds formed at the surface. The charge transfer mechanism is different in 
the $\mathrm{Ce}_{3} \mathrm{Pd}_{20} \mathrm{Si}_{6}$, occurring from $\mathrm{Si}$ (and $\mathrm{Ce}$ ) atoms towards more electronegative $\mathrm{Pd}$ atoms. The cages are also preserved at the surface but without the need for additional Ce guest surface atoms. Some electron localization is found at the position of the Si surface atoms but their Bader charge is found similar to that of corresponding bulk atoms. Therefore, the stabilization mechanism is slightly different in the two systems. Our results show that despite the Pd-based cage compound is clearly metallic, covalent-like interactions between Pd and $\mathrm{Si}$ atoms on the host cage structure significantly influences its surface structure. It leads

to a highly corrugated surface with a sub-nanometric periodicity of $a / \sqrt{2} \sim 8.8 \AA$ between protrusions consisting in $\mathrm{Pd} / \mathrm{Si}$ atomic ensembles. A parallel can be made with some of the Al-transition metals quasicrystalline approximants already mentioned in the Introduction, for which the cluster substructure can also be preserved at the surface, in relation to strong covalent-like interactions existing in the bulk.

\section{Conclusions}

We have performed a detailed investigation of the (100) surface of the $\mathrm{Ce}_{3} \mathrm{Pd}_{20} \mathrm{Si}_{6}$ cage compound to understand its relationship with the three-dimensional cage framework. The study has been carried out using both experimental surface science methods under ultrahigh vacuum conditions and theoretical calculations based on the Density Functional Theory (DFT). It is concluded that the surface forms at specific termination of the bulk structure, identified as layers of $\mathrm{Pd}_{12} \mathrm{Si}_{6}$ cages which are further stabilized by additional $\mathrm{Pd}$ atoms, rendering the surface termination more compact. The fact that the surface preserves the $\mathrm{Pd}_{12} \mathrm{Si}_{6}$ cages rather than other pure $\mathrm{Pd}$ cages suggests that they are more stable due to stronger bonding between $\mathrm{Si}$ and $\mathrm{Pd}$ atoms as revealed by the ELF calculations. It leads to a highly corrugated surface with a sub-nanometric periodicity that is directly linked to the cage framework. This approach thus provides an ultimate way for surface nanostructuration. Such surface structure investigations of cage compounds could be further 
complemented by either dynamical LEED or surface X-ray diffraction experiments. It would also be interesting also to extend such surface studies of intermetallic cage compounds as these self nano-structured surfaces might also have interesting chemical properties, either in the field of catalysis or potentially as hydrogen storage materials.

\section{Supporting Information}

Two-dimensional representations of the electron localization function calculated for different slab models. Calculated surface DOS for Pd1, Pd2 and Si atoms in model 2, compared to the corresponding bulk-like contributions in the slab.

\section{Acknowledgments}

High performance computing resources were provided by GENCI under Allocation 99642, as well as the EXPLOR center hosted by the Université de Lorraine (Allocation 2017M4XXX0108). EG acknowledges financial support through the COMETE project (COnception in silico de Matériaux pour l'EnvironnemenT et l'Energie) funded by the Lorraine Region. This work was also supported by the European C-MAC consortium and the French PIA project "Lorraine Université d'Excellence", reference ANR-15-IDEX-04-LUE.

\section{References}

(1) Rowe, D. Thermoelectrics Handbook: Macro to Nano; CRC Press, Taylor and Francis Group LLC, Boca Raton, 2006.

(2) Nolas, G.; Cohn, J.; Slack, G.; Schujman, S. Semiconducting Ge clathrates: Promising candidates for thermoelectric applications. Appl. Phys. Lett. 1998, 73, 178-180.

(3) Nolas, G.; Slack, G.; Schujman, S. Semiconductor clathrates: A phonon glass electron 
crystal material with potential for thermoelectric applications. Semiconduct. Semimet. 2001, 69, 255-300.

(4) Nolas, G.; Slack, G. Thermoelectric clathrates. Am. Sci. 2001, 89, 136-141.

(5) Takeda, N.; Kitagawa, J.; Ishikawa, M. New heavy-electron system $\mathrm{Ce}_{3} \mathrm{Pd}_{20} \mathrm{Si}_{6}$. J. Phys. Soc. Jpn. 1995, 64, 387-390.

(6) Gribanov, A.; Seropegin, Y.; Bodak, O. Crystal structure of the compounds $\mathrm{Ce}_{3} \mathrm{Pd}_{20} \mathrm{Ge}_{6}$ and $\mathrm{Ce}_{3} \mathrm{Pd}_{20} \mathrm{Si}_{6}$. J. Alloy. Compd. 1994, 204, L9- L11.

(7) Deen, P. P.; Strydom, A. M.; Paschen, S.; Adroja, D. T.; Kockelmann, W.; Rols, S. Quantum fluctuations and the magnetic ground state of $\mathrm{Ce}_{3} \mathrm{Pd}_{20} \mathrm{Si}_{6}$. Phys. Rev. B 2010, 81, 064427.

(8) Prokofiev, A.; Custers, J.; Kriegisch, M.; Laumann, S.; Mueller, M.; Sassik, H.; Svagera, R.; Waas, M.; Neumaier, K.; Strydom, A. M. et al. Crystal growth and composition-property relationship of $\mathrm{Ce}_{3} \mathrm{Pd}_{20} \mathrm{Si}_{6}$ single crystals. Phys. Rev. B 2009, $80,235107$.

(9) Custers, J.; Lorenzer, K.-A.; Mueller, M.; Prokofiev, A.; Sidorenko, A.; Winkler, H.; Strydom, A. M.; Shimura, Y.; Sakakibara, T.; Yu, R. et al. Destruction of the Kondo effect in the cubic heavy-fermion compound $\mathrm{Ce}_{3} \mathrm{Pd}_{20} \mathrm{Si}_{6}$. Nat. Mater. 2012, 11, 189-194.

(10) Yamaoka, H.; Schwier, E.; Arita, M.; Shimada, K.; Tsujii, N.; Jarrige, I.; Jiang, J.; Hayashi, H.; Iwasawa, H.; Namatame, H. et al. Electronic structure of the heavy-fermion caged compound $\mathrm{Ce}_{3} \mathrm{Pd}_{20} \mathrm{X}_{6}(\mathrm{X}=\mathrm{Si}, \mathrm{Ge})$ studied by density functional theory and photoelectron spectroscopy. Phys. Rev. B 2015, 91, 115139.

(11) Ono, H.; Nakano, T.; Takeda, N.; Ano, G.; Akatsu, M.; Nemoto, Y.; Goto, T.; Doenni, A.; Kitazawa, H. Magnetic phase diagram of clathrate compound $\mathrm{Ce}_{3} \mathrm{Pd}_{20} \mathrm{X}_{6}$ with quadrupolar ordering. J. Phys. Condens. Matter 2013, 25. 
(12) Dubois, J.; Belin-Ferré, E. Complex Metallic Alloys: Fundamentals and Applications; John Wiley \&Sons Inc., 2011.

(13) Thiel, P. A. Quasicrystal surfaces. Annu. Rev. Phys. Chem. 2008, 59, 129-152.

(14) Sharma, H.; Shimoda, M.; Tsai, A. Quasicrystal surfaces: structure and growth of atomic overlayers. Adv. Phys. 2007, 56, 403-464.

(15) Ledieu, J.; Gaudry, E.; Fournée, V. Surfaces of Al-based complex metallic alloys: atomic structure, thin film growth and reactivity. Sci. Technol. Adv. Mat. 2014, 15, 034802.

(16) Papadopolos, Z.; Kasner, G.; Ledieu, J.; Cox, E.; Richardson, N.; Chen, Q.; Diehl, R.; Lograsso, T.; Ross, A.; McGrath, R. Bulk termination of the quasicrystalline fivefold surface of $\mathrm{Al}_{70} \mathrm{Pd}_{21} \mathrm{Mn}_{9}$. Phys. Rev. B 2002, 66 .

(17) Sharma, H.; Fournée, V.; Shimoda, M.; Ross, A.; Lograsso, T.; Tsai, A.; Yamamoto, A. Structure of the fivefold surface of the icosahedral Al-Cu-Fe quasicrystal: Experimental evidence of bulk truncations at larger interlayer spacings. Phys. Rev. Lett. 2004, 93, 165502.

(18) Sharma, H.; Shimoda, M.; Sagisaka, K.; Takakura, H.; Smerdon, J. A.; Nugent, P. J.; McGrath, R.; Fujita, D.; Ohhashi, S.; Tsai, A. Structure of the fivefold surface of the Ag-In-Yb icosahedral quasicrystal. Phys. Rev. B 2009, 80, 121401(R).

(19) Ledieu, J.; Gaudry, E.; Loli, L. N. S.; Villaseca, S. A.; de Weerd, M.-C.; Hahne, M.; Gille, P.; Grin, Y.; Dubois, J.-M.; Fournée, V. Structural investigation of the (010) surface of the $\mathrm{Al}_{13} \mathrm{Fe}_{4}$ catalyst. Phys. Rev. Lett. 2013, 110, 076102.

(20) Scheid, P.; Chatelier, C.; Ledieu, J.; Fournee, V.; Gaudry, E. Bonding network and stability of clusters: the case study of the $\mathrm{Al}_{13} \mathrm{TM}_{4}$ pseudo-10fold surfaces. Acta Cryst. A 2019, 75, 49-52. 
(21) Anand, K.; Allio, C.; Krellner, C.; Nguyen, H.; Baitinger, M.; Grin, Y.; Ledieu, J.; Fournée, V.; Gaudry, E. Charge balance controls the (100) surface structure of the $\mathrm{Ba}_{8} \mathrm{Au}_{5.25} \mathrm{Ge}_{40.75}$ clathrate. J. Phys. Chem. C 2018, 122, 2215-2220.

(22) Anand, K.; Nguyen, H.; Baitinger, M.; Allio, C.; Krellner, C.; Grin, Y.; Ledieu, J.; Fournée, V.; Gaudry, E. Ba $\mathrm{Au}_{5.25} \mathrm{Ge}_{40.75}(110)$ : A nano-caged surface electronically controlled by Barium and Gold adatoms. J. Phys. Chem. C 2018, 122, 29298-29306.

(23) Armbruester, M.; Schloegl, R.; Grin, Y. Intermetallic compounds in heterogeneous catalysis-a quickly developing field. Sci. Technol. Adv. Mat. 2014, 15, 034803.

(24) Kameoka, S.; Xu, Y.; Nishimura, C.; Tsai, A. P. Special issue on advanced metallic materials for catalysis. Preface. Mater. Trans. 2015, 56, 459.

(25) Tsai, A. P.; Kameoka, S.; Nozawa, K.; Shimoda, M.; Ishii, Y. Intermetallic: A pseudoelement for catalysis. Acc. Chem. Res. 2017, 50, 2879-2885.

(26) Kojima, T.; Kameoka, S.; Fujii, S.; Ueda, S.; Tsai, A.-P. Catalysis-tunable Heusler alloys in selective hydrogenation of alkynes: A new potential for old materials. Sci. Adv. 2018, 4 .

(27) Armbruester, M.; Kovnir, K.; Friedrich, M.; Teschner, D.; Wowsnick, G.; Hahne, M.; Gille, P.; Szentmiklosi, L.; Feuerbacher, M.; Heggen, M. et al. $\mathrm{Al}_{13} \mathrm{Fe}_{4}$ as a low-cost alternative for Palladium in heterogeneous hydrogenation. Nat. Mater. 2012, 11, 690693.

(28) Wolf, R.; Lee, M.; Davis, R.; Fay, P.; Ray, J. Pressure-composition isotherms for Palladium hydride. Phys. Rev. B 1993, 48, 12415-12418.

(29) Chan, K.; Miller, M.; Peng, X. First-principles computational study of hydrogen storage in silicon clathrates. Mater. Res. Lett. 2017, 6, 72-78. 
(30) Kresse, G.; Hafner, J. Ab initio molecular dynamics for liquid metals. Phys. Rev. B $1993,47,558-561$.

(31) Kresse, G.; Hafner, J. Ab initio molecular-dynamics simulation of the liquid-metalamorphous-semiconductor transition in Germanium. Phys. Rev. B 1994, 49, 1425114269.

(32) Kresse, G.; Furthmüller, J. Efficiency of ab-initio total energy calculations for metals and semiconductors using a plane-wave basis set. Comput. Mater. Sci 1996, 6, 15 50.

(33) Kresse, G.; Furthmüller, J. Efficient iterative schemes for ab initio total-energy calculations using a plane-wave basis set. Phys. Rev. B 1996, 54, 11169-11186.

(34) Kresse, G.; Joubert, D. From ultrasoft pseudopotentials to the projector augmentedwave method. Phys. Rev. B 1999, 59, 1758-1775.

(35) Blöchl, P. E. Projector augmented-wave method. Phys. Rev. B 1994, 50, 17953-17979.

(36) Perdew, J.; Burke, K.; Ernzerhof, M. Generalized gradient approximation made simple. Phys. Rev. Lett. 1996, 77, 3865-3868.

(37) Perdew, J.; Burke, K.; Ernzerhof, M. Generalized gradient approximation made simple. Phys. Rev. Lett. 1997, 78, 1396-1396.

(38) Tersoff, J.; Hamann, D. R. Theory and application for the scanning tunneling microscope. Phys. Rev. Lett. 1983, 50, 1998-2001.

(39) Tersoff, J.; Hamann, D. R. Theory of the scanning tunneling microscope. Phys. Rev. B 1985, 31, 805-813.

(40) Moll, N.; Kley, A.; Pehlke, E.; Scheffler, M. GaAs equilibrium crystal shape from first principles. Phys. Rev. B 1996, 54, 8844-8855. 
(41) Bechstedt, F. Principles of Surface Physics; Springer, Berlin, 2003.

(42) Schmidt, W. III-V compound semiconductor (001) surfaces. Appl. Phys. A 2002, 75, 89.

(43) Kitchin, J.; Reuter, K.; Scheffler, M. Alloy surface segregation in reactive environments: First-principles atomistic thermodynamics study of $\mathrm{Ag}_{3} \mathrm{Pd}(111)$ in oxygen atmospheres. Phys. Rev. B 2008, 77, 075437.

(44) Capaz, R.; Dias, L.; Leitão, A.; Blum, R.-P.; Niehus, H.; Achete, C. Chemical identification in the $\mathrm{Cu}_{3} \mathrm{Au}(100)$ surface using scanning tunneling microscopy and first-principles calculations. Surf. Sci. 2007, 601, 5540-5545.

(45) Jenkins, S. Ternary half-metallics and related binary compounds: stoichiometry, surface states, and spin. Phys. Rev. B 2004, 70, 245401.

(46) Henkelman, G.; Arnaldsson, A.; Jónsson, H. A fast and robust algorithm for Bader decomposition of charge density. Comput. Mater. Sci 2006, 36, 354-360.

(47) Sanville, E.; Kenny, S.; Smith, R.; Henkelman, G. Improved grid-based algorithm for Bader charge allocation. J. Comp. Chem. 2007, 28, 899-908.

(48) Tang, W.; Sanville, E.; Henkelman, G. A grid-based Bader analysis algorithm without lattice bias. J. Phys. Condens. Matter 2009, 21, 084204.

(49) Yu, M.; Trinkle, D. Accurate and efficient algorithm for Bader charge integration. J. Chem. Phys. 2011, 134, 064111.

(50) Savin, A.; Becke, A.; Flad, J.; Nesper, R.; Preuss, H.; von Schnering, H. A new look at electron localization. Angew. Chem. Int. Ed. 1991, 30, 409-412.

(51) Kohout, M.; Savin, A. Atomic shell structure and electron numbers. Int. J. Quantum Chem. 1997, 60, 875-882. 
(52) Kozlowski, D.; Pilmé, J. New insights in quantum chemical topology studies using numerical grid-based analyses. J. Comp. Chem. 2011, 32, 3207-3217.

(53) Santis, L. D.; Resta, R. Electron localization at metal surfaces. Surf. Sci. 2000, 450, $126-132$.

(54) Becke, A.; Edgecombe, K. A simple measure of electron localization in atomic and molecular systems. J. Chem. Phys. 1990, 92, 5397-5403.

(55) Momma, K.; Izumi, F. VESTA: a three-dimensional visualization system for electronic and structural analysis. J. Appl. Crystallogr. 2008, 41, 653-658.

(56) Hüfner, S. Photoelectron Spectroscopy: Principles and Applications; Springer-Verlag, Berlin Heidelberg GmbH, 1995.

(57) Jeong, I.-K.; Darling, T.; Graf, M.; Heffner, R.; Lee, Y.; Vogt, T.; Jorgensen, J. D. Ternary half- metallics and related binary compounds: Stoichiometry, surface states, and spin. Phys. Rev. Lett. 2004, 92, 105702.

(58) Casadei, M.; Ren, X.; Rinke, P.; Rubio, A.; Scheffler, M. Density functional theory study of the $\alpha-\gamma$ phase transition in Cerium: Role of electron correlation and $f$-orbital localization. Phys. Rev. B 2016, 93, 075153.

(59) Mo, Y.; Tang, H.; Bansil, A.; Tao, J. Accurate lattice geometrical parameters and bulk moduli from a semilocal density functional. AIP Advances 2018, 8, 095209.

(60) Donohue, J. The Structure of the Elements; John Wiley \& Sons Inc., 1974.

(61) Tran, F.; Karsai, F.; Blaha, P. Nonmagnetic and ferromagnetic fcc Cerium studied with one-electron methods. Phys. Rev. B 2014, 89, 155106.

(62) Kittel, C. Introduction to Solid State Physics; John Wiley \& Sons Inc., 2005. 
(63) Janthon, P.; Luo, S.; Kozlov, S. M.; Viñes, F.; Limtrakul, J.; Truhlar, D.; Illas, F. Bulk properties of transition metals: A challenge for the design of universal density functionals. J. Chem. Theory Comput. 2014, 10, 3832-3839.

(64) Haas, P.; Tran, F.; Blaha, P. Calculation of the lattice constant of solids with semilocal functionals. Phys. Rev. B 2009, 79, 085104.

(65) Okada, Y.; Tokumaru, Y. Precise determination of lattice parameter and thermal expansion coefficient of silicon between 300 and 1500 K. J. Appl. Phys. 1984, 56, 314-320.

(66) Paier, J.; Marsman, M.; Hummer, K.; Kresse, G.; Gerber, I. C.; Ángyán, J. G. Screened hybrid density functionals applied to solids. J. Chem. Phys. 2006, 124, 154709.

(67) Mattsson, A.; Armiento, R.; Paier, J.; Kresse, G.; Wills, J.; Mattsson, T. The AM05 density functional applied to solids. J. Chem. Phys. 2008, 128, 084714.

(68) Portnichenko, P. Y.; Cameron, A. S.; Surmach, M. A.; Deen, P.; Paschen, S.; Prokofiev, A.; Mignot, J.; Strydom, A.; Telling, M.; Podlesnyak, A. et al. Momentumspace structure of quasielastic spin fluctuations in $\mathrm{Ce}_{3} \mathrm{Pd}_{20} \mathrm{Si}_{6}$. Phys. Rev. B 2015, 91, 094412 .

(69) Hofer, W.; Foster, A.; Shluger, A. Theories of scanning probe microscopes at the atomic scale. Rev. Mod. Phys. 2003, 75, 1287-1331.

(70) Tran, R.; Xu, Z.; Radhakrishnan, B.; Winston, D.; Sun, W.; Persson, K.; Ong, S. Surface energies of elemental crystals. Sci. Data 2016, 3, 160080.

(71) Matar, S.; Pöttgen, R. Chemical bonding in equiatomic cerium intermetallics - The case of CeMgSn, CePdSn and CeMgPb. Solid State Sciences 2015, 48, 205-211.

(72) Pauling, L. The nature of the chemical bond. IV. The energy of single bonds and the relative electronegativity of atoms. J. Am. Chem. Soc. 1932, 54, 3570-3582. 
(73) Pauling, L. The Nature of the Chemical Bond, 3rd ed.; Cornell University Press: Ithaca, NY, 1960. 


\section{TOC Graphic}
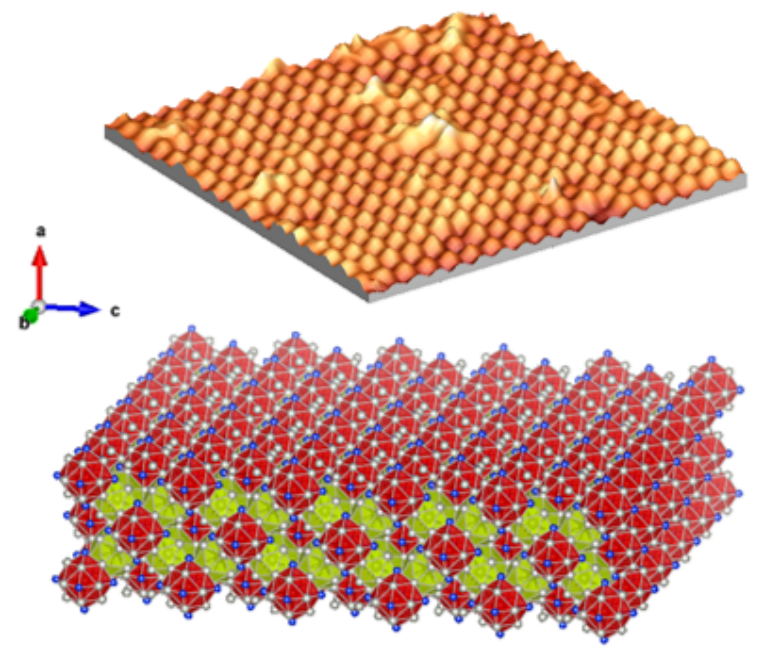